\title{
ScIDice
}

\author{
International Journal of Dentistry and Oral Science (IJDOS) \\ ISSN: 2377-8075
}

\section{Validation Of Indigenously Developed Video For Teaching Toothbrushing To Children With Autism Spectrum Disorder}

Research Article

Sakshi Chawla ${ }^{1 *}$, Mousumi Goswami

${ }^{1}$ Post Graduate Student (MDS), Department Of Paediatric \& Preventive Dentistry, ITS Dental College, Hospital \& Research Centre, Greater Noida.

${ }^{2}$ Prof \& Head, Department of Paediatric \& preventive dentistry, ITS Dental College, Hospital \& Research Centre, Greater Noida, India.

\section{Abstract}

\begin{abstract}
Aim: Validation of indigenously developed video for teaching toothbrushing to children with autism spectrum disorder. Methodology: This prospective pilot study was carried out in 10 participants of age 5-12 years. The developed video was taught to incorporate in the routine and transferred in parents smartphones after assessing their baseline descriptives, plaque index , toothbrushing efficiency and time required for perform the activity. The intention was to assess the improvement in toothbrushing technique and the ability of the child to comprehend and follow the instruction as shown in the video. Results: In first visit, most of the children with ASD were incapable of grasping the toothbrush properly and had irregular toothbrushing practices. The mean plaque score (Loe and Silness) has improved from $2.04 \pm 0.65$ to $2.03 \pm 0.12$ ( $p>0.05$ ) in the second visit and significantly improved from the baseline to third visit $(\mathrm{p}<0.05)$. Parental perception also highlighted improved compliance towards toothbrushing than before. The children showed less reluctance for toothbrushing which was a common concern for all the 10 set of parents.

Conclusion: Videos provides more comprehensible and structured way to deliver information and teach the desired action for the audience. Various task can be taught to children with ASD which may help children to cope better in dental environments during treatment.
\end{abstract}

\section{Introduction}

Learning in 21st century without technology is a setback for lot of individuals. Recommendations by the American Academy of Paediatric Dentistry suggests individualised preventive strategies should be unique to the need of the individual [1]. Considering the use of technological advancements and its interplay with success of health education. It becomes important to invest in tools that makes the teaching a lot more easier and comprehensible fora differently abled child.

Children diagnosed Autism spectrum disorder (ASD) experiences impaired social interactions and communication, also they faces restrictive and repetitive patterns of behaviour due to the neurodevelopmental dysfunctions [2]. Many of the elementary tasks like toothbrushing, constructions of sentences, making eye contact are delayed in this group. Visual Strategies in the form of photographs with auditory cues, individual task supports, visual scripts, video modelling aids in assisting in daily routines and thereby help improvement in the behaviour [3].

As many children with ASD are categorized as visual learners, their understanding is heightened as their visual processing skills are more than their audio or verbal processing $[4,5]$. Most commonly used technique is the Picture Exchange Communication System (PECS) which uses sticked pictures and written words that helps in teaching the skill of intentional communication to children as advocated by Heflin \& Alaimo in 2007 [6].

Visual pedagogy in the form of video provides a more structured and live instructional teaching that can be learned by the patient. It is also a part of education and treatment tool of autistic and related communication-handicapped children [7]. Video modelling is a method where an individual is asked to watch a given video to teach a desired skill. The targeted skill can be modelled by a peer, an adult or by themselves. The instructions are prompted and reinforced on the individual after which the person imitates the behaviour of the model as displayed in the video $[8,9]$.

\section{*Corresponding Author:}

Dr. Sakshi Chawla,

Post Graduate Student (MDS), Department Of Paediatric \& Preventive Dentistry, ITS Dental College, Hospital \& Research Centre, Greater Noida, India

Tel: +91-9711120059

E-mail: sakshichawla21@yahoo.co.in

Received: March 30, 2021

Accepted: June 20, 202

Published: June 25, 2021

Citation: Sakshi Chawla, Mousumi Goswami. Validation Of Indigenously Developed Video For Teaching Toothbrushing To Children With Autism Spectrum Disorder. Int J Dentistry Oral Sci. 2021;8(6):2828-2832. doi: http://dx.doi.org/10.19070/2377-8075-21000552

Copyright: Sakshi Chawla 2021 . This is an open-access article distributed under the terms of the Creative Commons Attribution License, which permits unrestricted use, distribution and reproduction in any medium, provided the original author and source are credited. 
Thus, this study intend to deliver video modelled toothbrushing social story with the help of indigenously created and validated video, modelled by a professional to teach correct and structured toothbrushing.

\section{Material And Methods}

This prospective pilot study was carried out in the department of paediatric and preventive dentistry. The study was ethically approved by the Institutional Review Board- I.T.S ethical committee [ITSDCGN/2018/001] and written informed consent were signed by all parents. The selected age group was 5-12 years with 4 female and 6 male participants. The intention was to assess the improvement in toothbrushing technique and the ability of the child to comprehend and follow the instruction as shown in the video. The success of which can be employed on researches carried out for large scale studies.

The ten child volunteers diagnosed with autism were attending a special school near the hospital.It was a volunteer based participation which did not included any financial or incentive support. The basic requirement of the study was aany smartphone with internet connectivity.

\section{Inclusion criteria:}

1. Having access to a mobile smartphone

2. Age group within 5-12 years

\section{Exclusion criteria:}

1. Highly uncooperative children during dental check-up.

2. Under medication affecting oral hygiene.

For this study, the widely advisedFones technique with horizontal scrub in the occlusal surfaces was used as the standard for proper toothbrushing technique. Fones technique is commonly suggested for young children with limited manual dexterity or motor coordination as it involve circular stroke on each set of teeth $[10,11]$. In addition to toothbrushing it also allows good gingival stimulation ensuring its health [12].

The video demonstration of Brushing technique was recorded that illustrated simple and structured steps such as Fill mug with water, Put paste on brush, Circles in front, Circle in right, Circle left, Clean the uppers, Scrub lowers, Clean the inners, Rinse and spit. The Audio was dubbed by recording it in parts in both Language -English and Hindi. Created video was then mixed, edited and modified as per to the responses from the one psychiatrist and a special educator. The final version was kept in MP4 format ensuring its compatibility by checking it in Android and Apple smartphones.[Included in supplementary material]

The study protocol was as follows. Baseline oral hygiene assessment by filled in WHO 2013 form, plaque index was recorded and frequency, agent, and technique of toothbrushing was noted.

At baseline and prior to the intervention, a brief demonstration of the created video was shown to the children in presence of parents, caretaker or teachers. The participants were made to watch video showing adult-modelled tooth brushing technique. Children were asked to use their regular toothbrush and toothpaste as used earlier. Training was provided to the parents record the toothbrushing activity for the follow-up evaluation.

The plaque score was assessed by running the explorer on the labial and lingual surfaces of the six indexed teeth. The modification was followed in case of any missing indexed teeth. A re-evaluation for created video demonstration was done after 1 day, on the 14th day and 30th day. After baseline the plaque assessment was recorded on 30th day of the intervention.

The caretaker/parents and teacher were asked not to modify the toothbrushing teaching apart from the technique shown in the video. However, support in the form of reinforcing the steps shown in the video was allowed in case any child needed additional help.

Toothbrushing ability was evaluated in the follow up by following Shin and Saeed [13] criteria. The seven main steps of evaluation : holding the tooth brush, brushing in circular motion on front teeth, upper teeth, lower teeth, inner teeth , rinsing and spitting the remaining toothpaste was scored as $0=$ inability to perform the step, Score $1=$ parent completed the step for the individual, Score $2=$ a prompt was required for the child to complete the step whereas Score 3 was given to children completed the step on their own. The mean score in the three recalls were documented and analysed. Finally, for each attempt on re-evaluation the length of time spent on Toothbrushing was also recorded in seconds.

\section{Analytical Approach}

Data was entered into Microsoft Excel spreadsheet and then checked for any missing entries. It was analysed using Statistical Package for Social Sciences (SPSS) version 21. Categorical variables were summarized as absolute $\&$ relative frequencies and continuous variables were summarized as mean and standard deviation.Graphs were prepared on Microsoft Excel.

Normality of the continuous variables was checked by Shapiro Wilk test. Data was found to be normal. Thus, inferential statistics were performed using parametric tests of significance.

Comparison of categorical variables was done using Chi square test. Intergroup comparison of continuous variables was done using Independent $t$ test. The level of statistical significance was set at 0.05 .

\section{Results}

The selected age group was 5-12 years with 4 female and 6 male participants having a mean age of $8.3 \pm 0.23$ years. The children had a prior diagnosis of Autism Spectrum Disorder recorded in their school records. Table 1 represents the descriptive data and baseline statistics collected at the baseline.

Effective brushing was evaluated based on simple 7 steps that involved brushing at the surfaces, rinsing and spitting. Mean score of each step from the participants was pictorially represented in Figure 1 across different timr frame (baseline $/ \mathrm{T}_{0}, \mathrm{~T}_{1}$ and $\mathrm{T}_{2}$ ). 
Table 1. Descriptive data of the baseline evaluation.

\begin{tabular}{|c|c|c|}
\hline & Frequency & Percentage \\
\hline Gender & Male $=6$, Female $=4$ & $60 \%, 40 \%$ \\
\hline Mean age & $8.3 \pm 0.23$ years & - \\
\hline Children brushing twice daily & 2 & $20 \%$ \\
\hline Children requiring assistance while brushing & 10 & $100 \%$ \\
\hline Children visiting/visited dentist before & 2 & $20 \%$ \\
\hline Children undergoing dental treatment & 0 & $0 \%$ \\
\hline Children spending 2 minutes for Brushing & 0 & $0 \%$ \\
\hline Baseline Mean Plaque Score & $2.04 \pm 0.65$ & - \\
\hline Baseline Mean Brushing Time & $1.01 \pm 0.57$ & - \\
\hline
\end{tabular}

Table 2. Mean plaque score and mean tooth brushing time assessed at T0,T1\& T2 time interval.

\begin{tabular}{|c|c|c|c|}
\hline & T0 & T1 & T2 \\
\hline Mean Plaque Score & $2.04 \pm 0.65$ & $2.03 \pm 0.12$ & $2.00 \pm 0.33$ \\
\hline Mean Brushing Time (min) & $1.01 \pm 0.57$ & $1.5 \pm 0.11$ & $1.6 \pm 0.09$ \\
\hline
\end{tabular}

Figure 1. Mean score of the toothbrushing steps evaluated at three different time intervals.

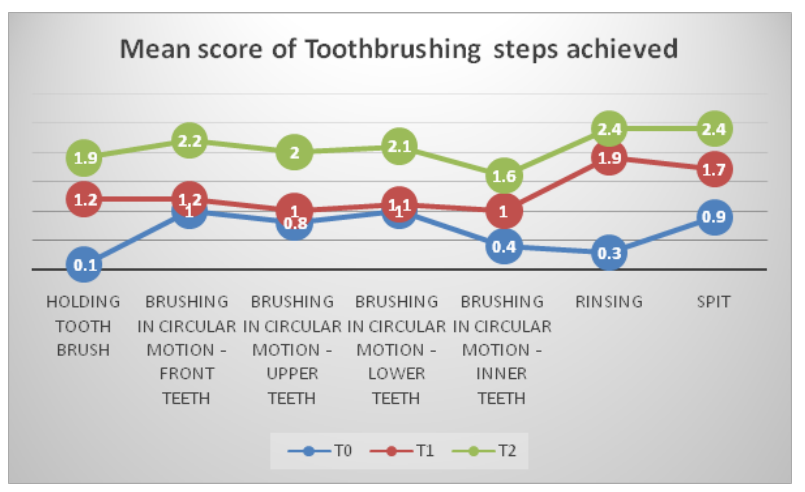

Mean plaque score at different time frame and the average time required for toothbrushing after watching the video was also noted as shown in table 2 .

\section{Discussion}

Children with ASD faces enormous challenges for dental care access and oral healthcare practices. Optimum oral care maintenance in children with ASD should to be of prime focus, since the existing dental problems may worsen and intensify, thereby impacting the individual's overall quality of life [14]. Thus, a holistic approach to oral care in children with ASD is substantially required [15].

The creation and validation of an educative video in case of an autistic childfor toothbrushing is relevant because it provides a pedagogic resource tool which can be offered to the maximum number of children for an independent and flexible learning. An educative video will address to the basic aspects related to the daily oral care for individual with autism, it is a promising effort to enhance home based oral health care practices and may also help children to cope during dental visits. The objective of the study was to validate a created educational video for children with autism and their families who experience difficulty in performing tooth brushing.
Such methodological intervention is being widely constructed, validated and used in many fields even by children with autism, aiming tostrengthen and stimulate the audience helping them to cope, sensitizeand learn new care strategies [16-19]. Instructive recordings are planned not exclusively to give new information, yet additionally to fortify existing ones by reinforcing as many times as needed by the patient or their families [20].

In spite of the thorough scientific literature based on the video modelling as an educative tool, the validation of any created video stand important both by the children and their parents for its effectivity, scientific anchoring and creditability.

In first visit, most of the children with ASD were incapable of grasping the toothbrush properly and had irregular toothbrushing practices. As per to parents/caretakers, 100\% children required assistance in performing tooth brushing and $80 \%$ were not practicing toothbrushing twice daily. The hurdled toothbrushing was indicating the difficulty faced by these children due to the fine and gross motor impairments, mental disabilities, and sensory problems, as well as the requirement of parent/caregiver support as reported by previous studies [21-25]. There were very few children who had visited a dentist before. Less awareness and low priority of dental care was a major factor for the poor oral health by researchers. It has been previously documented that children with ASD usually suffers from gingivitis, periodontitis, and dis- 
comfort owing to poor oral hygiene practices $[26,27]$.

The mean plaque score (Loe and Silness) 28 though non-significant but has improvedfrom $2.04 \pm 0.65$ to $2.03 \pm 0.12$ in the second visit and significantly improved from the baseline to third visit $(\mathrm{p}<0.05)$. The frequency of the brushing had improved as 4 children started brushing twice daily after 15 days of intervention with increased to 6 on the $30^{\text {th }}$ day of evaluation. This result was partially in agreement with Charlop-Christy et al [29] who observed the acquisition of the task in 5 children only after just 2 sessions of video modelling.

The ability to perform toothbrushing was also assessed by analysing seven different steps required to complete fones brushing technique. The mean score by the shin and saeed criteria depicted improvement at the third visit after practicing the toothbrushing with the video. The results were statistically significant $(p<0.05)$.

Parental perception also highlighted improved compliance towards toothbrushing than before. The children showed less reluctance for toothbrushing which was a common concern for all the 10 set of parents. The parental perception for the effectiveness of the created video reported both improved behaviour and acquisition of tooth brushing. One child displayed independent toothbrushing after 30 days of the intervention, the independency achieved was with the video when played simultaneously.

Although some results were not statistically significant, but the findings are promising. Consistently decreasing plaque scores over the course of the intervention, along with parental report of intervention success, suggest that the created video is a probable candidate for better toothbrushing practice.

According to the evaluation, the constructed video enhances interest in the activityand shortens skill acquisition time. Similar findings were observed in a researches carries out by CharlopChristy MH [29] , Keith D. Allen and Dustin P [30], MacDonald $\mathrm{R}$ and Clark M [31], Rehfeldt R and Dahman D [32].

This pilot study for the video validation was conducted with children from one special school diagnosed with the spectrum disorder. It is estimable that the obtained results justifies the developed video, being necessary to use as a resource for health education, evaluating its educational effectiveness.

\section{Conclusion}

Videos provides more comprehensible and structured way to deliver information and teach the desired action for the audience. It is also worth noting that the construction and validation of the educational video, with correct techniquecontributes for instructions based independent learning and performing the desired task even in the absence of health professionals/instructors.

\section{References}

[1]. American Academy of Pediatric Dentistry. Behavior guidance for the pediatric dental patient. The Reference Manual of Pediatric Dentistry. Chicago, Ill.: American Academy of Pediatric Dentistry; 2020:292-310.

[2]. Edition F. Diagnostic and statistical manual of mental disorders. Am Psychiatric Assoc. 2013;21.

[3]. Rao SM, Gagie B. Learning through seeing and doing: Visual supports for children with autism. Teaching exceptional children. 2006 Jul;38(6):26-33.

[4]. Santangelo SL, Tsatsanis K. What is known about autism: genes, brain, and behavior. Am J Pharmacogenomics. 2005;5(2):71-92. PubmedPMID: 15813671.

[5]. Mesibov GB, Shea V. The TEACCH program in the era of evidence-based practice. J Autism DevDisord. 2010 May;40(5):570-9. PubmedPMID: 19937103.

[6]. Heflin J, Alaimo DF. Students with autism spectrum disorders: Effective instructional practices. Recording for the Blind \& Dyslexic; 2007.

[7]. Bäckman B, Pilebro C. Visual pedagogy in dentistry for children with autism. ASDC J Dent Child. 1999 Sep-Oct;66(5):325-31, 294. PubmedPMID: 10631888.

[8]. Graetz JE, Mastropieri MA, Scruggs TE. Show time: Using video self-modeling to decrease inappropriate behavior. Teaching exceptional children. 2006 May;38(5):43-8.

[9]. Sigafoos J, O'Reilly M, De La Cruz B. How to use video modeling and video prompting. Pro-Ed; 2007.

[10]. Luppanapornlarp S, Leelataweewud P, Putongkam P, Ketanont S. Periodontal status and orthodontic treatment need of autistic children. World J Orthod. 2010 Fall;11(3):256-61. PubmedPMID: 20877735.

[11]. Ainamo J, Xie Q, Ainamo A, Kallio P. Assessment of the effect of an oscillating/rotating electric toothbrush on oral health. A 12-month longitudinal study. J ClinPeriodontol. 1997 Jan;24(1):28-33. PubmedPMID: 9049794.

[12]. Poyato-Ferrera M, Segura-Egea JJ, Bullón-Fernández P. Comparison of modified Bass technique with normal toothbrushing practices for efficacy in supragingival plaque removal. Int J Dent Hyg. 2003 May;1(2):110-4. PubmedPMID: 16451532.

[13]. Shin CJ, Saeed S. Toothbrushing barriers for people with developmental disabilities: a pilot study. Spec Care Dentist. 2013 Nov-Dec;33(6):269-74. PubmedPMID: 24164224

[14]. Yashoda R, Puranik MP. Oral health status and parental perception of child oral health related quality-of-life of children with autism in Bangalore, India. Journal of Indian Society of Pedodontics and Preventive Dentistry. 2014 Apr $1 ; 32(2): 135$

[15]. Norderyd J, Klingberg G, Faulks D, Granlund M. Specialised dental care for children with complex disabilities focusing on child's functioning and need for general anaesthesia. DisabilRehabil. 2017 Dec;39(24):2484-2491. PubmedPMID: 27871192.

[16]. Popple B, Wall C, Flink L, Powell K, Discepolo K, Keck D, et al. Brief Report: Remotely Delivered Video Modeling for Improving Oral $\mathrm{Hy}$ giene in Children with ASD: A Pilot Study. J Autism DevDisord. 2016 Aug;46(8):2791-2796. PubmedPMID: 27106570.

[17]. MacDonald R, Clark M, Garrigan E, Vangala M. Using video modeling to teach pretend play to children with autism. Behavioral Interventions: Theory \& Practice in Residential \& Community-Based Clinical Programs. 2005 Nov;20(4):225-38.

[18]. Rehfeldt RA, Dahman D, Young A, Cherry H, Davis P. Teaching a simple meal preparation skill to adults with moderate and severe mental retardation using video modeling. Behavioral Interventions: Theory \& Practice in Residential \& Community-Based Clinical Programs. 2003 Jul;18(3):209-18.

[19]. Aldi C, Crigler A, Kates-McElrath K, Long B, Smith H, Rehak K, et al. Examining the Effects of Video Modeling and Prompts to Teach Activities of Daily Living Skills. Behav Anal Pract. 2016 May 20;9(4):384-388. PubmedPMID: 27920969.

[20]. Ibrahim M, Antonenko PD, Greenwood CM, Wheeler D. Effects of segmenting, signalling, and weeding on learning from educational video. Learning, media and technology. 2012 Sep 1;37(3):220-35.

[21]. Dawson G, Watling R. Interventions to facilitate auditory, visual, and motor integration in autism: a review of the evidence. J Autism DevDisord. 2000 Oct;30(5):415-21. PubmedPMID: 11098877.

[22]. Stein LI, Polido JC, Mailloux Z, Coleman GG, Cermak SA. Oral care and sensory sensitivities in children with autism spectrum disorders. Spec Care Dentist. 2011 May-Jun;31(3):102-10. PubmedPMID: 21592164.

[23]. Stein LI, Polido JC, Cermak SA. Oral care and sensory concerns in autism. Am J OccupTher. 2012 Sep-Oct;66(5):e73-6. PubmedPMID: 22917131.

[24]. Stein LI, Polido JC, Cermak SA. Oral care and sensory over-responsivity in children with autism spectrum disorders. Pediatr Dent. 2013 MayJun;35(3):230-5. PubmedPMID: 23756306.

[25]. Kuhaneck HM, Chisholm EC. Improving dental visits for individuals with autism spectrum disorders through an understanding of sensory processing. Spec Care Dentist. 2012 Nov-Dec;32(6):229-33. PubmedPMID: 23095065.

[26]. Kopycka-Kedzierawski DT, Auinger P. Dental needs and status of autistic children: results from the National Survey of Children's Health. Pediatr Dent. 2008 Jan-Feb;30(1):54-8. PubmedPMID: 18402100.

[27]. Abdolsamadi HR, Vahedi M, Esmaeili F, Nazari S, Abdollahzadeh S. Serum Interleukin-6 as a Serologic Marker of Chronic Periapical Lesions: A Case- 
control Study. J Dent Res Dent Clin Dent Prospects. 2008 Spring;2(2):43-7. PubmedPMID: 23289057.

[28]. SILNESS J, LOE H. PERIODONTAL DISEASE IN PREGNANCY. II. CORRELATION BETWEEN ORAL HYGIENE AND PERIODONTAL CONDTION. ActaOdontol Scand. 1964 Feb;22:121-35. PubmedPMID: 14158464 .

[29]. Charlop-Christy MH, Le L, Freeman KA. A comparison of video modeling with in vivo modeling for teaching children with autism. J Autism DevDisord. 2000 Dec;30(6):537-52. PubmedPMID: 11261466.

[30]. Allen KD, Wallace DP, Renes D, Bowen SL, Burke RV. Use of video modeling to teach vocational skills to adolescents and young adults with autism spectrum disorders. Education and Treatment of Children. 2010;33(3):33949.

[31]. MacDonald R, Clark M, Garrigan E, Vangala M. Using video modeling to teach pretend play to children with autism. Behavioral Interventions: Theory \& Practice in Residential \& Community-Based Clinical Programs. 2005 Nov;20(4):225-38.

[32]. Rehfeldt RA, Dahman D, Young A, Cherry H, Davis P. Teaching a simple meal preparation skill to adults with moderate and severe mental retardation using video modeling. Behavioral Interventions: Theory \& Practice in Residential \& Community-Based Clinical Programs. 2003 Jul;18(3):209-18. 